\title{
Natural leptogenesis and neutrino masses with two Higgs doublets
}

\author{
Jackson D. Clarke, Robert Foot, and Raymond R. Volkas \\ ARC Centre of Excellence for Particle Physics at the Terascale, School of Physics, \\ University of Melbourne, Melbourne, Victoria 3010, Australia \\ (Received 14 June 2015; published 17 August 2015)
}

\begin{abstract}
The minimal Type I seesaw model cannot explain the observed neutrino masses and the baryon asymmetry of the Universe via hierarchical thermal leptogenesis without ceding naturalness. We show that this conclusion can be avoided by adding a second Higgs doublet with $\tan \beta \gtrsim 4$. The models considered naturally accommodate a standard model-like Higgs boson and predict TeV-scale scalar states and low- to intermediate-scale hierarchical leptogenesis with $10^{3} \mathrm{GeV} \lesssim M_{N_{1}} \lesssim 10^{8} \mathrm{GeV}$.
\end{abstract}

DOI: 10.1103/PhysRevD.92.033006

PACS numbers: $14.60 . \mathrm{Pq}, 14.60 . \mathrm{St}, 14.80 . \mathrm{Ec}$

\section{INTRODUCTION}

The discovery of a neutral Higgs boson at the LHC $[1,2]$ has strengthened the case for the standard model (SM) paradigm of spontaneous electroweak symmetry breaking: a scalar doublet $\Phi$ gains a vacuum expectation value (vev) $\langle\Phi\rangle=v / \sqrt{2} \approx 174 \mathrm{GeV}$ by virtue of the potential

$$
V_{\mathrm{SM}}=\mu^{2} \Phi^{\dagger} \Phi+\lambda\left(\Phi^{\dagger} \Phi\right)^{2}
$$

with $\mu^{2}<0$. As well, the measurement of neutrino oscillations [3-8] suggests that the SM should be extended to incorporate neutrino masses. A straightforward way to achieve this is to include three right-handed neutrinos. Then gauge invariance allows two extra renormalizable terms to be added to the Yukawa Lagrangian,

$$
-\Delta \mathcal{L}_{Y}=\left(y_{\nu}\right)_{i j} \bar{l} \bar{l}_{L}^{i} \tilde{\Phi} \nu_{R}^{j}+\frac{1}{2} M_{i} \overline{\left(\nu_{R}^{i}\right)^{c}} \nu_{R}^{i}+\text { H.c. }
$$

where $l_{L}=\left(\nu_{L}, e_{L}\right)^{T}, \tilde{\Phi}=i \tau_{2} \Phi^{*}$, and $M_{i}$ are the righthanded neutrino masses. The SM extended in this way is what we refer to as the minimal Type I seesaw model [9-12].

If $y_{\nu} v \ll M_{i}$ then the minimal Type I seesaw provides an elegant explanation for the smallness of the neutrino masses. After electroweak symmetry breaking, the neutrino mass matrix is given by the seesaw formula

$$
m_{\nu}=\frac{v^{2}}{2} y_{\nu} \mathcal{D}_{M}^{-1} y_{\nu}^{T}
$$

where $\mathcal{D}_{M} \equiv \operatorname{diag}\left(M_{1}, M_{2}, M_{3}\right)$, suppressed by the presumably large right-handed neutrino mass scale.

The minimal Type I seesaw also provides a mechanism to reproduce the baryon asymmetry of the Universe (BAU). Fukugita-Yanagida hierarchical thermal leptogenesis [13] proceeds via the $C P$-violating out-of-equilibrium decays of the lightest right-handed neutrino $N_{1}$, creating a lepton asymmetry which is reprocessed into the baryon sector by the electroweak sphalerons. Successful hierarchical thermal leptogenesis is possible when the Davidson-Ibarra bound (ensuring enough $C P$ asymmetry in the decays) is satisfied $[14,15]$,

$$
M_{N_{1}} \gtrsim 5 \times 10^{8} \mathrm{GeV}\left(\frac{v}{246 \mathrm{GeV}}\right)^{2}
$$

where $v$ is the vev that enters the seesaw of Eq. (3).

The ability of the minimal Type I seesaw model to simultaneously explain neutrino masses and the BAU is certainly intriguing. However, Vissani observed [16] that the model is incapable of doing so without generating a naturalness problem. ${ }^{1}$ Equation (4) is simply incompatible with the conservative naturalness requirement that corrections to the electroweak $\mu^{2}$ parameter of Eq. (1) not exceed $1 \mathrm{TeV}^{2}$. With three flavors of hierarchical right-handed neutrinos, this requires [17]

$$
M_{N_{1}} \lesssim 3 \times 10^{7} \mathrm{GeV}\left(\frac{v}{246 \mathrm{GeV}}\right)^{\frac{2}{3}}
$$

The incompatibility is exemplified in Fig. 1; nowhere at $v=246 \mathrm{GeV}$ is it possible to simultaneously fulfill the Davidson-Ibarra and Vissani bounds.

A sensible question is then: in what minimal ways can this incompatibility be overcome? Figure 1 suggests three conspicuous (but not mutually exclusive) options: (1) Modify the correction to $\mu^{2}$, e.g., by restoring supersymmetry or by partly cancelling the correction from the heavy fermion loop [18,19]; (2) lower the Davidson-Ibarra bound, e.g., by considering resonant leptogenesis [20], an alternative mechanism [21], or by introducing new fields which allow an increased $C P$ asymmetry in the righthanded neutrino decay; (3) seek an extension of the canonical seesaw for neutrino mass, i.e., reduce the (possibly effective) $v$ entering the seesaw Eq. (3).

In this paper we will consider the third option. Specifically we will examine alternative seesaw possibilities when the minimal Type I see-saw model is extended

\footnotetext{
${ }^{1}$ Naturalness is admittedly an aesthetic requirement of a model, and the possibility remains that nature is just fine-tuned.
} 


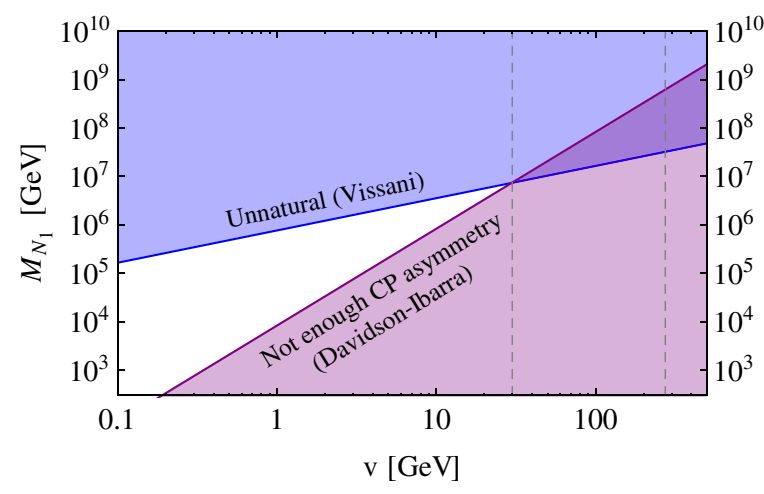

FIG. 1 (color online). Bounds on hierarchical thermal leptogenesis as a function of $v$. Shown is the Davidson-Ibarra bound (purple) and the Vissani bound (blue). The dashed lines indicate $v=246,30 \mathrm{GeV}$.

by a second Higgs doublet $\Phi_{2}$. We are motivated by the following observation: if the seesaw neutrino mass of Eq. (3) is evaluated at $v \lesssim 30 \mathrm{GeV}$, then Eqs. (4) and (5) become compatible, as is clear from Fig. 1. Thus, we expect that two-Higgs-doublet models with right-handed neutrinos ( $\nu 2 \mathrm{HDMs}$ ) and $\tan \beta=v_{1} / v_{2} \gtrsim 8$, where $\Phi_{2}$ is responsible for a tree-level seesaw, can naturally accommodate leptogenesis and neutrino masses. In fact, we find that $\tan \beta \gtrsim 4$ is possible, since the extra scalar states can be naturally $\mathrm{TeV}$ scale and the Vissani bound can be relaxed.

Upon examining the $22 \mathrm{HDM}$ scenarios which will succeed, we rediscover the radiative Ma model [22] as the only possibility when $v_{2}=0$. Otherwise, in models without a significant radiative neutrino mass component, we require $0.3 \lesssim v_{2} / \mathrm{GeV} \lesssim 60$. The potentially small vev is made natural by softly breaking a $U(1)$ or $Z_{2}$ symmetry, which also automatically results in one SM-like $C P$-even state. One advantage of this scenario is that it can work in all $\nu 2$ HDM types, greatly increasing the opportunity for model building.

The paper is organized as follows. In Sec. II we build the 2 2HDM models of interest, describe the scalar states, and briefly review the relevant experimental constraints. In Sec. III we pay particular attention to naturalness limits on the extra scalars; we verify that a natural $\nu 2 \mathrm{HDM}$ of any type is still allowed by experiment. We discuss neutrino masses in Sec. IV and leptogenesis in Sec. V. The region of parameter space which naturally achieves hierarchical leptogenesis is identified. We conclude in Sec. VI.

\section{II. $\nu$ 2HDM MODEL}

\section{A. Lagrangian}

The scalar content of the model contains two doublets $\Phi_{1,2}$ each with hypercharge +1 . For simplicity we consider
TABLE I. The four models with no tree-level flavor-changing neutral currents and allowing for a $\mathrm{GeV}$-scale vev to provide the seesaw while preserving perturbativity of $y_{t}$.

\begin{tabular}{lllll}
\hline \hline Model & $u_{R}^{i}$ & $d_{R}^{i}$ & $e_{R}^{i}$ & $\nu_{R}^{i}$ \\
\hline Type I & $\Phi_{1}$ & $\Phi_{1}$ & $\Phi_{1}$ & $\Phi_{2}$ \\
Type II & $\Phi_{1}$ & $\Phi_{2}$ & $\Phi_{2}$ & $\Phi_{2}$ \\
LS & $\Phi_{1}$ & $\Phi_{1}$ & $\Phi_{2}$ & $\Phi_{2}$ \\
Flipped & $\Phi_{1}$ & $\Phi_{2}$ & $\Phi_{1}$ & $\Phi_{2}$ \\
\hline \hline
\end{tabular}

the softly broken, $C P$-conserving, $Z_{2}$-symmetric potential (see, e.g., Ref. [23])

$$
\begin{aligned}
V_{2 \mathrm{HDM}}= & m_{11}^{2} \Phi_{1}^{\dagger} \Phi_{1}+m_{22}^{2} \Phi_{2}^{\dagger} \Phi_{2}-m_{12}^{2}\left(\Phi_{1}^{\dagger} \Phi_{2}+\Phi_{2}^{\dagger} \Phi_{1}\right) \\
& +\frac{\lambda_{1}}{2}\left(\Phi_{1}^{\dagger} \Phi_{1}\right)^{2}+\frac{\lambda_{2}}{2}\left(\Phi_{2}^{\dagger} \Phi_{2}\right)^{2} \\
& +\lambda_{3}\left(\Phi_{1}^{\dagger} \Phi_{1}\right)\left(\Phi_{2}^{\dagger} \Phi_{2}\right)+\lambda_{4}\left(\Phi_{1}^{\dagger} \Phi_{2}\right)\left(\Phi_{2}^{\dagger} \Phi_{1}\right) \\
& +\frac{\lambda_{5}}{2}\left[\left(\Phi_{1}^{\dagger} \Phi_{2}\right)^{2}+\left(\Phi_{2}^{\dagger} \Phi_{1}\right)^{2}\right],
\end{aligned}
$$

where all the parameters are real. To explain observations, at least one of these doublets must obtain a nonzero vev. We consider $m_{11}^{2}<0$ and a $C P$-conserving vacuum,

$$
\left\langle\Phi_{1}\right\rangle_{0}=\frac{1}{\sqrt{2}}\left(\begin{array}{c}
0 \\
v_{1}
\end{array}\right), \quad\left\langle\Phi_{2}\right\rangle_{0}=\frac{1}{\sqrt{2}}\left(\begin{array}{c}
0 \\
v_{2}
\end{array}\right),
$$

where $v_{1}>0, v_{2} \geq 0$, and $v_{1}^{2}+v_{2}^{2}=v^{2} \approx(246 \mathrm{GeV})^{2}$.

A general $2 \mathrm{HDM}$ will have flavor-changing neutral currents at tree level. These can be avoided if right-handed fermions of a given type $\left(u_{R}^{i}, d_{R}^{i}, e_{R}^{i}\right)$ couple to only one of the doublets $[24,25]$. Although not strictly necessary, we will assume that this is realized and adopt the convention that only $\Phi_{1}$ couples to the $u_{R}^{i}$. In a $\nu 2 \mathrm{HDM}$, if we assume this also applies for the $\nu_{R}^{i}$, then there are eight possibilities. As mentioned in the Introduction, the seesaw constraint Eq. (3) can be made consistent with naturalness and leptogenesis if the vev contributing to the seesaw is sufficiently small. Since we would like our model to remain perturbative, and already $y_{t} \approx 1$ for $v \approx 246 \mathrm{GeV}$, we anticipate that $\Phi_{2}$ obtains the small vev, and thus we couple it to the $\nu_{R}^{i}$. Remaining are four possible $\nu 2$ HDMs which we refer to by their conventional types as listed in Table I. ${ }^{2}$ The Yukawa Lagrangian is then given by

$$
\begin{aligned}
-\mathcal{L}_{Y}= & +y_{u} \overline{q_{L}} \tilde{\Phi}_{1} u_{R}+y_{d} \overline{q_{L}} \Phi_{I} d_{R} \\
& +y_{e} \overline{l_{L}} \Phi_{J} e_{R}+y_{\nu} \bar{l}_{L} \tilde{\Phi}_{2} \nu_{R} \\
& +\frac{1}{2} M_{N} \overline{\left(\nu_{R}\right)^{c}} \nu_{R}+\text { H.c. }
\end{aligned}
$$

${ }^{2}$ Type I $\nu 2 \mathrm{HDMs}$ with $v_{2} \sim \mathrm{eV}$ were considered in Refs. [2629]. We will end up considering $v_{2}$ of $\mathcal{O}(0.1-10) \mathrm{GeV}$. 
where $I, J$ depend on the model type, and family indices are implied.

\section{B. Scalar masses and mixings}

Consistency with experiments requires the extra scalar states to have masses at least $\gtrsim 80 \mathrm{GeV}$. To construct models with potentially $\mathrm{TeV}$-scale scalars with a naturally small $v_{2}$, we will consider $m_{22}^{2}>0$ and $m_{12}^{2} / m_{22}^{2} \ll 1$ [26]. This is technically natural, since in the limit $m_{12}^{2} / m_{22}^{2} \rightarrow 0$ a $U(1)$ or $Z_{2}$ symmetry is restored if $\lambda_{5}=0$ or $\lambda_{5} \neq 0$, respectively.

For $m_{11}^{2}<0$, the vevs are given by

$$
\begin{gathered}
v_{1} \approx \sqrt{\frac{-2 m_{11}^{2}}{\lambda_{1}}}, \\
v_{2} \approx \frac{1}{1+\frac{v_{1}^{2}}{2 m_{22}^{2}} \lambda_{345}} \frac{m_{12}^{2}}{m_{22}^{2}} v_{1},
\end{gathered}
$$

where $\lambda_{345}=\lambda_{3}+\lambda_{4}+\lambda_{5}$. These relations become exact when $m_{12}^{2}=0$ and when $\lambda_{2}=0$, respectively. For $m_{22}^{2} \gg \lambda_{345} v_{1}^{2}, \tan \beta \equiv v_{1} / v_{2} \approx m_{22}^{2} / m_{12}^{2}$.

There is a useful constraint on $m_{22}^{2}$ which is derived as follows. The minimization conditions give

$$
m_{22}^{2}+\frac{1}{2} \lambda_{2} v_{2}^{2}=\tan ^{2} \beta\left(m_{11}^{2}+\frac{1}{2} \lambda_{1} v_{1}^{2}\right),
$$

where $\lambda_{1} v_{1}^{2} \approx m_{h}^{2} \approx(125 \mathrm{GeV})^{2}$ (see below). In the limit $m_{22}^{2} \gg \lambda_{2} v_{2}^{2} / 2$, it can be seen that $m_{22}^{2}$ is bounded above by

$$
m_{22}^{2} \lesssim \frac{1}{2} m_{h}^{2} \tan ^{2} \beta
$$

if $m_{11}^{2}$ is to remain negative. Figure 2 illustrates how $m_{11}^{2}$ deviates from its standard value of $-(88 \mathrm{GeV})^{2}$ as $m_{22}^{2}$ approaches this bound. For $m_{22}^{2}$ above this bound, $m_{11}^{2}$ very quickly grows to values $>v^{2}$, and $v \approx 246 \mathrm{GeV}$ is only

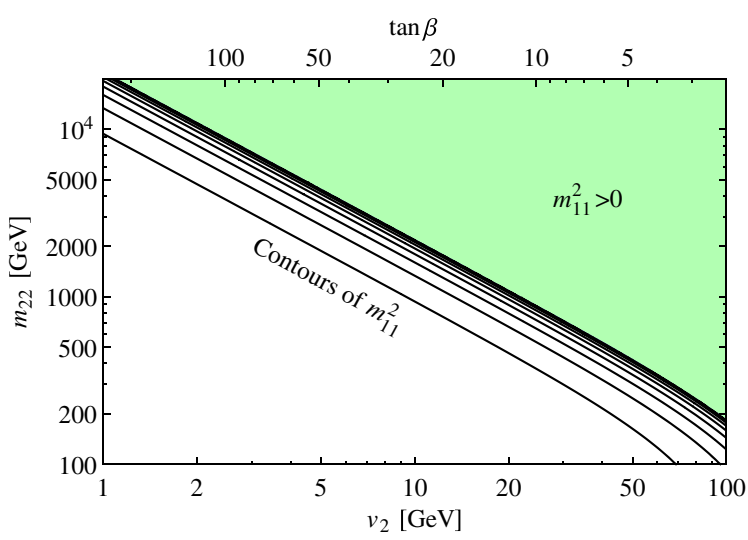

FIG. 2 (color online). Contours of $m_{11}^{2} / \mathrm{GeV}^{2}=-80^{2},-70^{2}$, and so on. The shaded region has no solution for $m_{11}^{2}<0$. explained by a miraculous balance of $m_{11}^{2}$ against $m_{22}^{2} / \tan ^{2} \beta$, which constitutes a fine-tuning. Thus, we adopt Eq. (12) as a consistency condition.

The charged scalar and pseudoscalar (neutral scalar) mass-squared matrices are diagonalized by a mixing angle $\beta(\alpha)$. The neutral mass eigenstates are

$$
\begin{aligned}
h & =\rho_{1} \cos \alpha+\rho_{2} \sin \alpha, \\
H & =\rho_{2} \cos \alpha-\rho_{1} \sin \alpha, \\
A & =\eta_{2} \sin \beta-\eta_{1} \cos \beta,
\end{aligned}
$$

where $\rho_{i}=\sqrt{2} \operatorname{Re}\left(\Phi_{i}^{0}\right)-v_{i}$ and $\eta_{i}=\sqrt{2} \operatorname{Im}\left(\Phi_{i}^{0}\right)$. The masses are given by

$$
\begin{aligned}
m_{h}^{2} & =\lambda_{1} v_{1}^{2}+\mathcal{O}\left(\frac{m_{12}^{4}}{m_{22}^{4}} v_{1}^{2}\right), \\
m_{H}^{2} & =m_{22}^{2}+\frac{1}{2} \lambda_{345} v_{1}^{2}+\mathcal{O}\left(\frac{m_{12}^{4}}{m_{22}^{4}} m_{22}^{2}\right), \\
m_{A}^{2} & =m_{22}^{2}+\frac{1}{2}\left(\lambda_{345}-2 \lambda_{5}\right) v_{1}^{2}+\mathcal{O}\left(\frac{m_{12}^{4}}{m_{22}^{4}} m_{22}^{2}\right), \\
m_{H^{ \pm}}^{2} & =m_{22}^{2}+\frac{1}{2} \lambda_{3} v_{1}^{2}+\mathcal{O}\left(\frac{m_{12}^{4}}{m_{22}^{4}} m_{22}^{2}\right),
\end{aligned}
$$

i.e., the same as in the inert doublet model [23] up to corrections proportional to $m_{12}^{4} / m_{22}^{4}$, which we provide in Appendix A. Clearly, if $m_{22}^{2} \gg v^{2}$, the mass scale of extra scalar states is $\approx m_{22}$.

In the alignment limit $\cos (\alpha-\beta) \rightarrow 0$, the couplings of $h$ to SM particles become SM-like. We calculate

$$
\begin{aligned}
& \cos ^{2}(\alpha-\beta) \\
& \approx \frac{m_{12}^{4}}{m_{22}^{4}} \frac{v_{1}^{4}}{m_{22}^{4}} \frac{\left(\lambda_{1}-\lambda_{345}\right)^{2}}{\left(1-\frac{v_{1}^{2}}{2 m_{22}^{2}}\left(2 \lambda_{1}-\lambda_{345}\right)\right)^{2}\left(1+\frac{v_{1}^{2}}{2 m_{22}^{2}} \lambda_{345}\right)^{2}},
\end{aligned}
$$

suppressed by the approximate $U(1)$ or $Z_{2}$ symmetry $\left(m_{12}^{2} / m_{22}^{2} \ll 1\right)$ as well as the usual decoupling limit suppression $\left(v_{1}^{2} / m_{22}^{2} \ll 1\right)$ [30]. Thus, the model naturally accommodates a SM-like neutral scalar state.

\section{Constraints}

With $M_{N}>m_{22}$ the constraints (and search strategies) for a $\nu 2 \mathrm{HDM}$ of a given type are largely identical to those for a $2 \mathrm{HDM}$ of the same type, for which there is extensive literature (see references henceforth). The 2HDM potential Eq. (6) is subject to a few standard theoretical constraints [23]. The necessary and sufficient conditions for positivity of the potential in all directions are [31-33]

$$
\begin{aligned}
\lambda_{1,2} & \geq 0, \\
\lambda_{3} & \geq-\sqrt{\lambda_{1} \lambda_{2}}, \\
\lambda_{3}+\lambda_{4}-\left|\lambda_{5}\right| & \geq-\sqrt{\lambda_{1} \lambda_{2}} .
\end{aligned}
$$


Vacuum stability of the potential minimum is more difficult to evaluate. An inequality which ensures a global minimum, missing possible metastable vacua, is presented in Ref. [34]. Tree-level perturbative unitarity of scalar-scalar scattering is ensured by bounding the eigenvalues of the scattering matrix [23,35-37]. Perturbativity of the $\lambda_{i}$ can also be demanded [38]. At the very least, these bounds should be implemented at the mass scale of the scalar states. In addition they may be demanded up to some high scale under the renormalization group evolution, which results in nontrivial constraints on the parameter space (see, e.g., Refs. [39-41] $]^{3}$ ). Type II, lepton-specific (LS), and flipped 2HDMs are particularly susceptible to exclusion by such a demand at large $\tan \beta$; at one loop, their Yukawa couplings hit a Landau pole before $M_{\mathrm{Pl}} \sim 10^{18} \mathrm{GeV}$ when $v_{2} \lesssim 3.6,2.3,3.3 \mathrm{GeV}(\tan \beta \gtrsim 68$, $107,75)$ respectively [42]. These Landau poles merely indicate the breakdown of perturbativy.

The scalar boson discovered at the LHC is to be identified with the mass eigenstate $h$. Its couplings have been measured to be SM-like, which constrains the $\nu 2 \mathrm{HDM}$ to lie in the alignment limit $\cos (\alpha-\beta) \approx 0$, particularly at large $\tan \beta$, and for Type II and flipped 2 HDMs. ${ }^{4}$ As is evident from Eq. (15), the alignment limit is automatically preferred in our model due to the approximate $Z_{2}$ or $U(1)$ symmetry. Thus, we limit the following discussion on additional experimental limits to those that constrain moderate to large $\tan \beta$ models very close to the alignment limit.

In Type II and flipped $2 \mathrm{HDMs}$, the $\Phi_{2}$ coupling to down-type quarks is $\tan \beta$ enhanced. The $H^{ \pm}$state then contributes significantly to radiative $B \rightarrow X_{s} \gamma$ decay; the experimental measurement [49] combined with a recent next-to-next-to-leading-order SM calculation [50] bounds $m_{H^{ \pm}} \gtrsim 480 \mathrm{GeV}$ at $95 \%$ C.L. for $\tan \beta \gtrsim 2$. This bound along with the consistency condition Eq. (12) implies $v_{2} \lesssim$ $45 \mathrm{GeV}(\tan \beta \gtrsim 5.4)$ for these $\nu 2 \mathrm{HDMs}$. In the Type II 2HDM, the $\Phi_{2}$ coupling to $e_{R}^{i}$ is also $\tan \beta$ enhanced, and the bound on $m_{H^{ \pm}}$from $B \rightarrow \tau \nu$ decays exceeds the radiative bound when $\tan \beta \gtrsim 60$ [23].

Direct searches at Large Electron-Positron Collider constrain $m_{H^{ \pm}} \gtrsim 80 \mathrm{GeV}$ assuming decay to SM particles [51]. At the LHC, searches for $H / A \rightarrow \tau \tau$ [52,53] are particularly constraining in the Type II $2 \mathrm{HDM}$. The 95\% C.L. limit rises approximately linearly from $m_{A} \gtrsim 300 \mathrm{GeV}$ at $\tan \beta=10$ to $m_{A} \gtrsim 1000 \mathrm{GeV}$ at $\tan \beta=60$. Such searches can also be mildly constraining for the LS $2 \mathrm{HDM}$ at moderate $\tan \beta$. Searches for $H^{ \pm} \rightarrow \tau \nu$ [54,55] cannot compete with $B \rightarrow X_{s} \gamma$ for Type II/flipped 2HDMs or with $H / A \rightarrow \tau \tau$ for the LS 2HDM. However, for $m_{H^{ \pm}}<160 \mathrm{GeV}$, significant parameter space is ruled out in Type I 2HDMs with moderate $\tan \beta$.

\footnotetext{
${ }^{3}$ Note that some of the bounds derived in these papers do not apply to the softly broken $Z_{2}$-symmetric case and also do not apply to the $Z_{2}$-symmetric case when one of the vevs vanishes.

${ }^{4}$ We refer the reader to Refs. [41,43-48] for allowed parameter space as a function of $\cos (\alpha-\beta)$ and $\tan \beta$ in all 2HDM types.
}

The $\left(y_{\nu}\right)_{i j} l_{L}^{i} \tilde{\Phi}_{2} \nu_{R}^{j} \quad$ Yukawa term related to the neutrino masses can induce lepton flavor violating decays; these are suppressed by the small $y_{\nu}$ and the right-handed neutrino mass scale $M_{N}>m_{22}$. The processes of interest are $l_{\alpha} \rightarrow l_{\beta} \gamma, l_{\alpha} \rightarrow 3 l_{\beta}$, and $\mu \rightarrow e$ conversion in nuclei (see Ref. [56] for expressions). As well, $b \rightarrow s l_{\alpha} \bar{l}_{\beta}$ decays are induced in Type II and flipped $\nu 2 \mathrm{HDMs}$. In practice, lepton flavor violating measurements constrain linear combinations of $\left(y_{\nu}\right)_{i j}$ bi- and trilinears as well as the $M_{N_{i}}$.

In summary, for moderate to large $\tan \beta$ and $\cos (\alpha-\beta) \approx 0$, experiments are most constraining for the Type II and flipped 2HDMs, with $m_{22} \gtrsim 480 \mathrm{GeV}$ necessary (implying $v_{2} \lesssim 45 \mathrm{GeV}$ ). For Type I and LS 2HDMs, even additional scalars with masses down to $80 \mathrm{GeV}$ may still have evaded detection.

\section{NATURALNESS}

In the SM, the renormalization group equation (RGE) for the electroweak $\mu$ parameter [as in Eq. (1)] is dominated by the top quark Yukawa,

$$
\frac{d \mu^{2}}{d \ln \mu_{R}} \approx \frac{1}{(4 \pi)^{2}} 6 y_{t}^{2} \mu^{2}
$$

where $\mu_{R}$ is the renormalization scale. At low energy we measure $\mu^{2} \approx-(88 \mathrm{GeV})^{2}$, and under $\mathrm{SM}$ running it is apparent that $|\mu|$ remains $\sim 100 \mathrm{GeV}$ even up to the Planck scale $M_{\mathrm{Pl}} \sim 10^{18} \mathrm{GeV}$. Thus, there is no measurable naturalness problem in the SM alone; there is no finetuning of any measurable parameter at a high scale, only the cancellation of an unmeasurable bare parameter against an unphysical cutoff scale, which should be assigned no physical significance. With this understood, it is clear that a measurable naturalness problem can only arise when $d \mu^{2} / d \ln \mu_{R} \gtrsim \mu^{2}$. Indeed, this is exactly how the Vissani bound in the Type I seesaw model can be interpreted $[16,17]$. Let us now examine when the $\nu 2 \mathrm{HDM}$ encounters such a problem.

In practice, the naturalness considerations can be divided into two distinct calculations: the influence of $m_{22}$ on $m_{11}$ and the influence of $M_{N}$ on $m_{22}$. These influences will be considered in turn. ${ }^{5}$

\section{A. Corrections to $m_{11}^{2}$}

If $m_{22}^{2} \ll m_{h}^{2} \tan ^{2} \beta / 2$, then $m_{11}^{2}$ sets the mass of the observed SM-like Higgs via Eqs. (9) and (14). The oneloop RGE for the $m_{11}^{2}$ parameter is [23] (see Ref. [41] for a recent two-loop calculation)

\footnotetext{
${ }^{5}$ In the following we ignore the influence of the small $y_{\nu}$ Yukawas on $m_{11}^{2}$, and hence those results also hold in a general 2HDM
} 


$$
\frac{d m_{11}^{2}}{d \ln \mu_{R}}=\frac{1}{(4 \pi)^{2}}\left[\left(4 \lambda_{3}+2 \lambda_{4}\right) m_{22}^{2}+\mathcal{O}\left(m_{11}^{2}\right)\right] .
$$

The $\mathcal{O}\left(m_{11}^{2}\right)$ term contains gauge, $\lambda_{1}$, and Yukawa contributions, which, as in the SM case, do not induce a naturalness problem. However, if $\lambda_{3,4}$ are nonzero, then a naturalness problem is induced for sufficiently large $m_{22}^{2}$; we are interested in when this generically occurs. Even if $\lambda_{3,4}=0$ at some scale, they will quickly be reintroduced by gauge interactions at one loop. Their one-loop RGEs are given by

$$
\begin{aligned}
& \frac{d \lambda_{3}}{d \ln \mu_{R}}=\frac{1}{(4 \pi)^{2}}\left[\frac{3}{4}\left(g_{Y}^{4}-2 g_{Y}^{2} g_{2}^{2}+3 g_{2}^{4}\right)+\cdots\right], \\
& \frac{d \lambda_{4}}{d \ln \mu_{R}}=\frac{1}{(4 \pi)^{2}}\left[3 g_{Y}^{2} g_{2}^{2}+\cdots\right],
\end{aligned}
$$

where $g_{2}^{2}\left(m_{Z}\right) \approx 0.43$ and $g_{Y}^{2}\left(m_{Z}\right) \approx 0.13$ are the gauge couplings and the ellipses contain terms multiplicative in $\lambda_{3,4}$, terms proportional to $\lambda_{5}^{2}$, and terms related to the Yukawas. Let us ignore those effects for now and return to them later. Note that ignoring the contribution from $\lambda_{5}^{2}$ is equivalent to assuming $\lambda_{5} \lesssim 0.2$, so that its contribution is subdominant to the gauge couplings. Typically, one would expect

$$
\begin{aligned}
\left|\lambda_{3}\left(\mu_{R}\right)\right| & \gtrsim \frac{1}{(4 \pi)^{2}} \frac{3}{4}\left(g_{Y}^{4}-2 g_{Y}^{2} g_{2}^{2}+3 g_{2}^{4}\right), \\
\left|\lambda_{4}\left(\mu_{R}\right)\right| & \gtrsim \frac{1}{(4 \pi)^{2}} 3 g_{Y}^{2} g_{2}^{2},
\end{aligned}
$$

and thus

$$
\left|\frac{d m_{11}^{2}}{d \ln \mu_{R}}\right| \gtrsim \frac{1}{(4 \pi)^{4}}\left(3 g_{Y}^{4}+9 g_{2}^{4}\right) m_{22}^{2} .
$$

This lower bound is of the same order as the two-loop pure gauge contribution [41].

Equation (21) represents a conservative bound on the running of the $m_{11}^{2}$ parameter above the scale $\sim m_{22}$. Naturalness demands that this running not be significantly larger than the value measured at a low scale, $\left|m_{11}\right| \approx 88 \mathrm{GeV}$. A very conservative naturalness bound is therefore

$$
\begin{gathered}
\frac{1}{(4 \pi)^{4}}\left(3 g_{Y}^{4}+9 g_{2}^{4}\right) m_{22}^{2}<1 \mathrm{TeV}^{2}, \\
\Rightarrow m_{22} \lesssim 1 \times 10^{5} \mathrm{GeV}
\end{gathered}
$$

Alternatively, we can try to bound a quantity which measures the fine-tuning in $m_{11}^{2}$ at some high scale $\Lambda_{h}$. A typical quantity is $[57,58]$

$$
\Delta\left(\Lambda_{h}\right)=\left|\frac{m_{11}^{2}\left(\Lambda_{h}\right)}{m_{11}^{2}(0)} \frac{\partial m_{11}^{2}(0)}{\partial m_{11}^{2}\left(\Lambda_{h}\right)}\right|,
$$

which compares percentage variations of two (in principle) measurable parameters. Let us now estimate how such a bound might constrain $m_{22}$.

For simplicity, and anticipating that the $m_{22}$ scale is not far above the electroweak scale, we will evolve the dimensionless parameters using the $(\nu) 2 \mathrm{HDM}$ RGEs from the $m_{Z}$ scale. First, the one-loop gauge coupling RGEs $[23,59]$ can be solved analytically. Upon substitution into the $\lambda_{3,4}$ RGEs [Eqs. (19)], and considering only the pure gauge contribution, the $\lambda_{3,4}$ running can be solved for given initial conditions. For simplicity we take $\lambda_{3}\left(m_{22}\right)=$ $\lambda_{4}\left(m_{22}\right) \equiv \lambda_{3,4}\left(m_{22}\right)$ and consider it a free parameter. Next we solve Eq. (18) for $m_{11}^{2}\left(\mu_{R}\right)$ with the initial condition $m_{11}^{2}\left(m_{22}\right)=-(88 \mathrm{GeV})^{2}$ (neglecting any RGE evolution of $\left.m_{22}^{2}\right)$. With these simplifications $\partial m_{11}^{2}(0) / \partial m_{11}^{2}\left(\Lambda_{h}\right)=1$, and the fine-tuning measure is given simply by $\Delta\left(\Lambda_{h}\right)=\left|m_{11}^{2}\left(\Lambda_{h}\right) /(88 \mathrm{GeV})^{2}\right|$.

Note that in setting the initial condition $m_{11}^{2}\left(m_{22}\right)=$ $-(88 \mathrm{GeV})^{2}$ we have implicitly assumed that $m_{22}^{2} \ll$ $m_{h}^{2} \tan ^{2} \beta / 2$ [see Eq. (12) and Fig. 2]. This is conservative for negative $m_{11}^{2}$, since $\left|m_{11}^{2}\left(m_{22}\right)\right|$ shrinks as $m_{22}^{2} / \tan ^{2} \beta \rightarrow$ $m_{h}^{2} / 2$ and the naturalness constraint would become more stringent. In some circumstances we will obtain naturalness bounds on $m_{22}^{2}$ which exceed $m_{h}^{2} \tan ^{2} \beta / 2$, which just indicates that the naturalness constraint is weaker than the consistency condition Eq. (12).

In Fig. 3 we show $\Delta=10$ and $\Delta=100$ contours as a function of $\Lambda_{h}$ and $\lambda_{3,4}\left(m_{22}\right)$. These represent naturalness upper bounds on $m_{22}$. The cusplike structures of apparently low fine-tuning in $m_{11}^{2}$ occur when $m_{11}^{2}$ runs negative before turning and passing through $m_{11}^{2}=0$, which just corresponds to a fine-tuning in $\left(\lambda_{3,4}, \Lambda_{h}\right)$. A stringent naturalness constraint is obtained by demanding $\Delta<10$ at $\Lambda_{h}=M_{\mathrm{Pl}}$; from Fig. 3 it is clear that this implies

$$
m_{22} \lesssim \text { few } \times 10^{3} \mathrm{GeV} .
$$

If any new physics comes in below $M_{\mathrm{Pl}}$, then the running of $m_{11}^{2}$ could change, and these bounds do not apply. If that is the case, then it is more appropriate to consider $\Lambda_{h}$ at the scale of the new physics, which weakens the bound, as is clear from Fig. 3. In the $22 \mathrm{HDM}$ this new physics scale is the right-handed neutrino scale $M_{N}$, after which the righthanded neutrinos can contribute to the running of $m_{11}^{2}$ through $m_{22}^{2}$ at one loop.

We have so far ignored the RGE contributions from possibly large Yukawas. There are two situations in which the Yukawas play a significant role. The first is in Type II, LS, and flipped $\nu 2 \mathrm{HDMs}$ with $\tan \beta$ large enough such that an early Landau pole is induced (see Sec. II C), and the second is in Type II and flipped 22HDMs with 

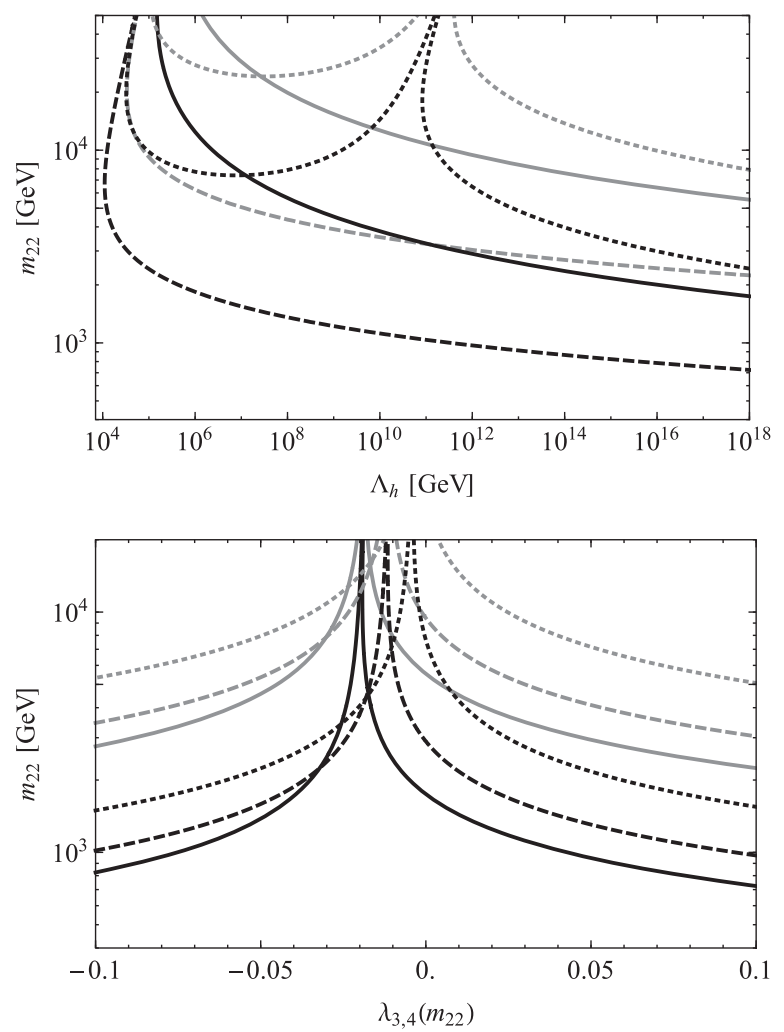

FIG. 3. Contours of the fine-tuning measure $\Delta\left(\Lambda_{h}\right)=10(100)$ in black (gray) as a function of (top) $\Lambda_{h}$ for $\lambda_{3,4}\left(m_{22}\right)=$ 0.0, 0.1, -0.01 (solid, dashed, dotted) and (bottom) $\lambda_{3,4}\left(m_{22}\right)$ for $\Lambda_{h}=10^{18}, 10^{12}, 10^{7} \mathrm{GeV}$ (solid, dashed, dotted). See the text for the assumptions that accompany this plot.

moderate to large $\tan \beta$ when the pure Yukawa term $\pm \frac{1}{(4 \pi)^{2}} 12 y_{b}^{2} y_{t}^{2}$ induced by a quark box diagram contributes significantly to the $\lambda_{3,4}$ RGEs [Eqs. (19)]. In Fig. 4 we show how the $\Delta=10$ contours change as a function of $v_{2}$ in an example Type II $\nu 2 \mathrm{HDM}$. For this figure we have numerically solved the full set of one-loop RGEs [41] including the top/bottom/tau Yukawas, taking the following values at the scale $M_{Z}: g_{s}^{2}=1.48, \lambda_{1}=\lambda_{2}=0.26$, and $y_{t}=0.96 / \sin \beta, \quad y_{b}=0.017 / \cos \beta, \quad y_{\tau}=0.010 / \cos \beta$. Comparing to Fig. 3 it can be seen that the pure Yukawa term has a noticeable effect when $v_{2} \lesssim 20 \mathrm{GeV}$. It is also apparent from Fig. 4 that nearing $v_{2} \approx 3.6 \mathrm{GeV}$ (below which a Landau pole is induced before $M_{\mathrm{Pl}}$ ) can act to degrade or improve the naturalness bound. The $v_{2}=3 \mathrm{GeV}$ bound in Fig. 4 shows the effect of hitting the Landau pole at $\sim 10^{9} \mathrm{GeV}$. We note that this only signals the breakdown of perturbation theory, and of our one-loop RGEs; we cannot calculate $m_{22}\left(\mu_{R}\right)$ above this scale, though it is perfectly possible that the theory remains natural.

In a repeated full one-loop RGE analysis, we found that the flipped $\nu 2 \mathrm{HDM}$ gave essentially the same results as the Type II $\nu 2 \mathrm{HDM}$ in Fig. 4 , and there was no noticeable Yukawa effect in the LS $\nu 2$ HDM until the Landau pole was
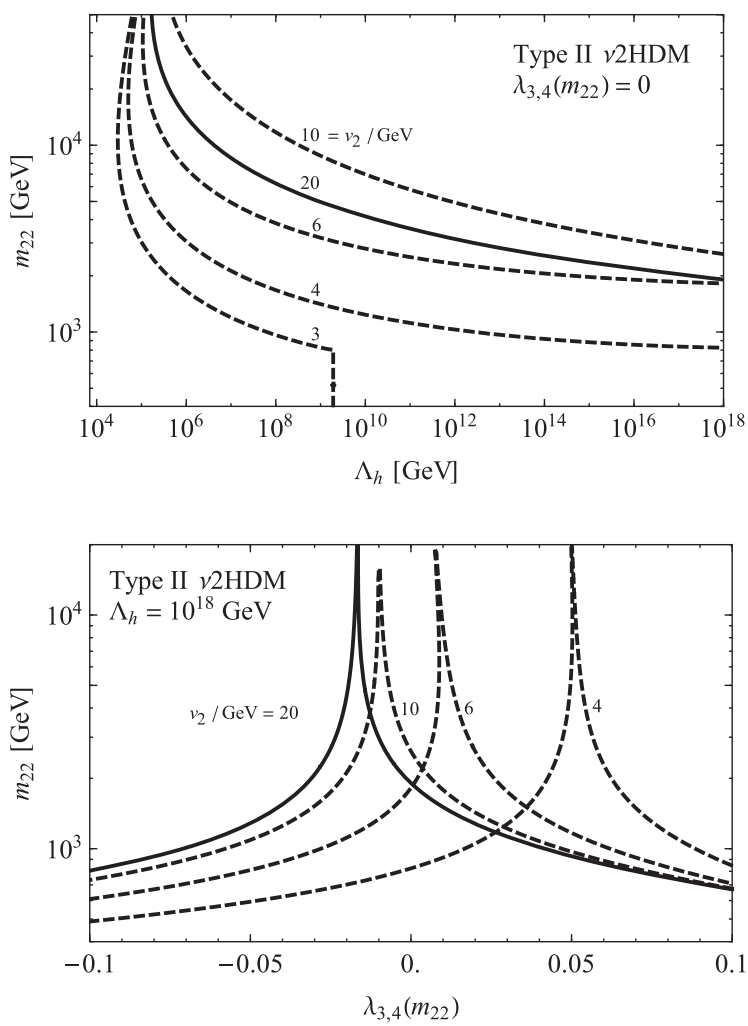

FIG. 4. Contours of the fine-tuning measure $\Delta\left(\Lambda_{h}\right)=10$ in an illustrative Type II $\nu 2 \mathrm{HDM}$ (see the text) for different values of $v_{2}$. The solid lines for $v_{2}=20 \mathrm{GeV}$ match approximately onto the solid lines in Fig. 3.

reached. Thus, we found that the stringent naturalness bounds of Eq. (25) and Fig. 3 are applicable at all times in the Type I $\nu 2 \mathrm{HDM}$, for $v_{2} \gtrsim 2 \mathrm{GeV}$ in the LS $\nu 2 \mathrm{HDM}$, and for $v_{2} \gtrsim 20 \mathrm{GeV}$ in the Type II and flipped $\nu 2 \mathrm{HDMs}$. Otherwise, Yukawa effects must be taken into account. Either way, the important point is now clear: a TeV-scale $m_{22}$ can be both completely natural and, as was discussed in the previous subsection, is experimentally allowed in all $\nu$ HDM types.

\section{B. Corrections to $\boldsymbol{m}_{22}^{2}$}

Let us now consider the influence of the right-handed neutrinos. The one-loop RGE for $m_{22}^{2}$ is $[17,60]$

$$
\frac{d m_{22}^{2}}{d \ln \mu_{R}}=\frac{1}{(4 \pi)^{2}}\left[-4 \operatorname{Tr}\left[y_{\nu} \mathcal{D}_{M}^{2} y_{\nu}^{\dagger}\right]+\mathcal{O}\left(m_{22}^{2}\right)\right]
$$

A conservative naturalness bound is obtained by bounding the running as we did in Eq. (23),

$$
\frac{1}{4 \pi^{2}} \operatorname{Tr}\left[y_{\nu} \mathcal{D}_{M}^{2} y_{\nu}^{\dagger}\right]<\Lambda_{\text {bound }}^{2},
$$

where taking $\Lambda_{\text {bound }}=1 \mathrm{TeV}$ gives the Vissani bound on $M_{N_{1}}$ of Eq. (5) [17]. However, now we are bounding corrections to $m_{22}^{2}$ rather than $m_{11}^{2}$, which may be TeV 


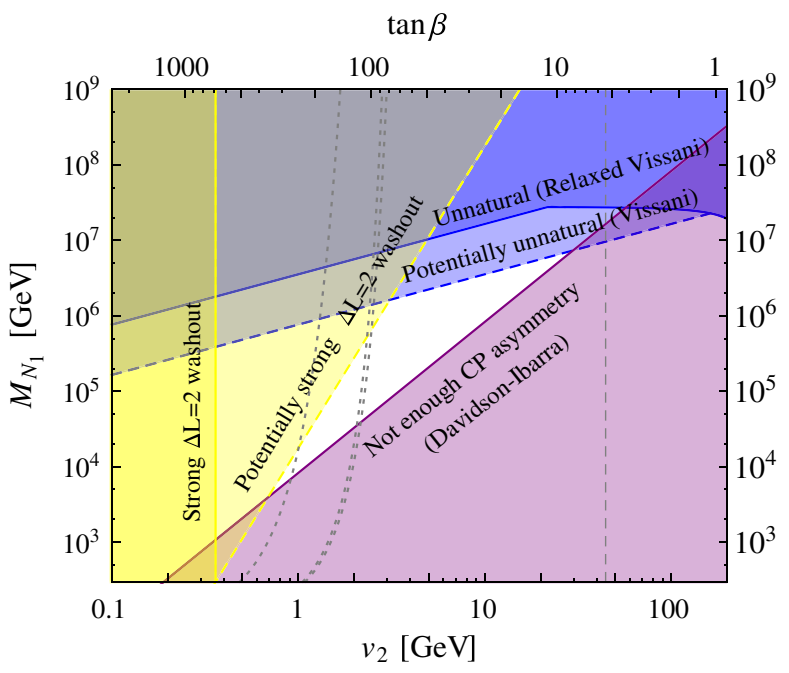

FIG. 5 (color online). Bounds on the $\nu 2 \mathrm{HDM}$ as a function of $v_{2}$. Shown (as labelled) are the Davidson-Ibarra bound, the Vissani and relaxed Vissani naturalness bounds, and the areas of parameter space with strong $\Delta L=2$ scattering washout. The Type II and flipped $\nu 2$ HDMs are excluded by $B \rightarrow X_{s} \gamma$ for values of $v_{2}$ greater than indicated by the gray dashed line (see Sec. II C). The gray dotted lines indicate the $v_{2}$ below which the Yukawas hit a Landau pole before $M_{N_{1}}$ in the Type II, flipped, and LS $\nu 2$ HDMs right to left.

scale. Thus, depending on the mass of the extra scalars, it is possible that we can sensibly take $\Lambda_{\text {bound }}>1 \mathrm{TeV}$, in which case the the naturalness bound is somewhat relaxed; in Fig. 5 we show a relaxed Vissani bound for $\Lambda_{\text {bound }}=\min \left(10 \mathrm{TeV}, 10 \sqrt{m_{h}^{2} \tan ^{2} \beta / 2}\right)$, where we have kept in mind the consistency condition Eq. (12). The Vissani bound still represents the unnatural area of parameter space if $m_{22}$ is closer to $100 \mathrm{GeV}$.

As before, we could instead bound a quantity which measures the fine-tuning in $m_{22}^{2}$ at some high scale $\Lambda_{h}$. In this case, the fine-tuning measure of Eq. (24) is

$\Delta\left(\Lambda_{h}\right)=1+\frac{1}{4 \pi^{2}} \frac{\sum_{i, j}\left(y_{\nu}\right)_{i j} M_{j}^{2}\left(y_{\nu}^{\dagger}\right)_{j i} \ln \left(M_{j} / \Lambda_{h}\right)}{m_{22}^{2}\left(M_{j}\right)}$.

Taking $m_{22}\left(M_{j}\right) \sim 1 \mathrm{TeV}$ and demanding $\Delta\left(M_{\mathrm{Pl}}\right)<10$ gives a similar bound to Vissani [Eq. (27) with $\left.\Lambda_{\text {bound }}=1 \mathrm{TeV}\right]$. Note that there is no naturalness bound on $M_{N}$ in the $y_{\nu} \rightarrow 0$ limit. This is the technically natural limit corresponding to an enhanced Poincaré symmetry in which $\nu_{R}$ decouples from the theory [61].

In summary, there are up to three scales in the $22 \mathrm{HDM}$ : $v, m_{22}$, and $M_{N}$. We have described the conditions under which $v^{2}$ (or $m_{11}^{2}$ ) is protected from $m_{22}^{2}$, and $m_{22}^{2}$ from $M_{N}^{2}$. Under such conditions it follows that $m_{11}^{2}$ is also protected from $M_{N_{1}}^{2}$ and the model is entirely natural.

\section{NEUTRINO MASSES}

If $m_{12}^{2}=0$ and $\lambda_{5}=0$, then a $U(1)$ lepton number symmetry can be defined, and neutrinos remain massless. Let us now consider turning each nonzero in turn.

$$
\text { A. } m_{12}^{2}>0, \lambda_{5}=0
$$

If $m_{12}^{2}>0$ then the $U(1)$ lepton number symmetry is softly broken; i.e., the breaking does not force us to insert a nonzero $\lambda_{5}$ term in order to introduce a divergent counterterm, and it is consistent to consider $m_{12}^{2}>0, \lambda_{5}=0$.

In this situation the neutrino mass matrix is given by the seesaw formula,

$$
m_{\nu}=\frac{v_{2}^{2}}{2} y_{\nu} \mathcal{D}_{M}^{-1} y_{\nu}^{T} \approx \frac{1}{\tan ^{2} \beta} \frac{v^{2}}{2} y_{\nu} \mathcal{D}_{M}^{-1} y_{\nu}^{T},
$$

where $\tan \beta \approx m_{22}^{2} / m_{12}^{2}$ for $m_{22}^{2} \gg \lambda_{345} v_{1}^{2}$ [see Eq. (10)].

The analogous Davidson-Ibarra and Vissani bounds are given by the standard Eqs. (4) and (5) with the replacement $v \rightarrow v_{2}$. These bounds are depicted in Fig. 5. If $v_{2} \lesssim$ $30 \mathrm{GeV}(\tan \beta \gtrsim 8)$, then both bounds are satisfied. As well, as discussed in Sec. III, if $m_{22}$ is TeV scale, the Vissani bound can be relaxed, and the required $C P$ asymmetry needed to reproduce the BAU via leptogenesis may be naturally achieved for $v_{2} \lesssim 60 \mathrm{GeV}(\tan \beta \gtrsim 4)$. In the Type I $\nu 2 \mathrm{HDM}, v_{2}$ can be naturally $\ll \mathrm{GeV}$. Otherwise, requiring a perturbative theory up to $M_{N_{1}}$ restricts $v_{2} \gtrsim 1 \mathrm{GeV}\left(v_{2} \gtrsim 2 \mathrm{GeV}\right)$ for the LS (Type II/ flipped) $\nu 2 \mathrm{HDM}$ in the parameter space region of interest, as depicted in Fig. 5.

$$
\text { B. } m_{12}^{2}=0, \lambda_{5} \neq 0
$$

In this situation $v_{2}=0$, and a $Z_{2}$ symmetry remains unbroken; this is the scenario of Ma [22]. The model yields a radiative neutrino mass and a dark matter candidate. This is only possible in the Type I $\nu 2 \mathrm{HDM}$, since in any other type the unbroken $Z_{2}$ forbids a Dirac mass term for any charged fermion coupling to $\Phi_{2}$. Note that the limit $\lambda_{5} \rightarrow 0$ is technically natural, since in that limit the $U(1)$ lepton number symmetry is reinstated.

If $M_{N}^{2} \gg m_{22}^{2}, v^{2}$ the radiatively induced neutrino mass matrix is

$$
\left(m_{\nu}\right)_{i j} \approx \frac{v^{2}}{2} \frac{\left(y_{\nu}\right)_{i k}\left(y_{\nu}^{T}\right)_{k j}}{M_{k}} \frac{\lambda_{5}}{8 \pi^{2}}\left(\ln \left[\frac{2 M_{k}^{2}}{\left(m_{H}^{2}+m_{A}^{2}\right)}\right]-1\right) .
$$

The analogous Davidson-Ibarra and Vissani bounds are given by the standard Eqs. (4) and (5) with the intuitive replacement $v^{2} \rightarrow v^{2} \frac{\lambda_{5}}{8 \pi^{2}}\left(\ln \left[2 M_{N_{1}}^{2} /\left(m_{H}^{2}+m_{A}^{2}\right)\right]-1\right)$. This assumes that there is no fine-tuning in the complex $y_{\nu}$ parameters to reproduce the observed neutrino masses (see Appendix B for details). These bounds are depicted in Fig. 6, where the Davidson-Ibarra bound has been 


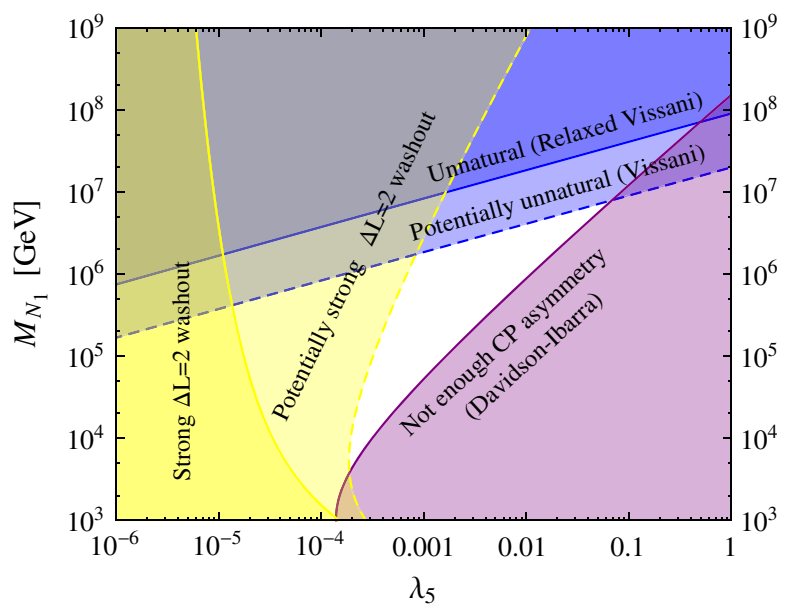

FIG. 6 (color online). As in Fig. 1 but for the Ma model. The Vissani and relaxed Vissani bounds are evaluated at $m_{22}=$ $100,1000 \mathrm{GeV}$ respectively. The Davidson-Ibarra bound and strong $\Delta L=2$ washout region are shown for $m_{22}=500 \mathrm{GeV}$, though they are only mildly sensitive to $m_{22}$.

evaluated at $m_{22}=500 \mathrm{GeV}$ as an illustrative example (the bound is only mildly sensitive to $m_{22}$ ). We find that the Ma model with $\lambda_{5} \lesssim 0.5$ can naturally achieve the required $C P$ asymmetry to reproduce the BAU via hierarchical leptogenesis. ${ }^{6}$

$$
\text { C. } m_{12}^{2}>0, \lambda_{5} \neq 0
$$

In this case both the tree-level seesaw and the radiative mechanism will contribute to the neutrino mass. Both contributions are calculable, and either might dominate. Note that it is still technically natural to take $\lambda_{5} \rightarrow 0$ in this case, since it restores a softly broken $U(1)$ symmetry. In other words, the $\lambda_{5}$ RGEs to all orders will be multiplicative in $\lambda_{5}$, indicative of the fact that the softbreaking term can only generate finite $U(1)$-breaking corrections.

\section{LEPTOGENESIS}

The observed BAU is achieved analogously to standard hierarchical thermal leptogenesis [13]; the out-ofequilibrium $C P$-violating decays of the lightest righthanded neutrino $N_{1} \rightarrow l \Phi_{2}$ create a lepton asymmetry which is transferred to the baryons by the electroweak sphalerons above $T \sim 100 \mathrm{GeV}$.

The details of the leptogenesis are largely defined by the decay parameter

$$
K=\frac{\Gamma_{D}}{\left.H\right|_{T=M_{1}}}=\frac{\tilde{m}_{1}}{m_{*}}
$$

\footnotetext{
${ }^{6} \mathrm{~A}$ similar observation was made in a recent paper [62].
}

comparing the rate for decays and inverse decays to the expansion rate at the time of departure from thermal equilibrium. Here, the rates

$$
\begin{gathered}
\Gamma_{D}=\frac{1}{8 \pi}\left(y_{\nu}^{\dagger} y_{\nu}\right)_{11} M_{1}, \\
H \approx \frac{17 T^{2}}{M_{\mathrm{Pl}}},
\end{gathered}
$$

are typically rescaled and expressed in terms of an effective neutrino mass $\tilde{m}_{1}$ and an equilibrium neutrino mass $m_{*}$,

$$
\begin{aligned}
& \tilde{m}_{1}=\frac{\left(y_{\nu}^{\dagger} y_{\nu}\right)_{11} v^{2}}{2 M_{1}}, \\
& m_{*} \approx 1.1 \times 10^{-3} \mathrm{eV}\left(\frac{v}{246 \mathrm{GeV}}\right)^{2},
\end{aligned}
$$

where $v$ is the vev that enters the seesaw Eq. (2). In the $\nu 2 \mathrm{HDM}$ with $\lambda_{5}=0$ (with $m_{12}^{2}=0, \lambda_{5} \neq 0$ ), the analogous definitions make the replacement $v^{2} \rightarrow v_{2}^{2}$ $\left(v^{2} \rightarrow v^{2} \frac{\lambda_{5}}{8 \pi^{2}}\left(\ln \left[2 M_{N_{1}}^{2} /\left(m_{H}^{2}+m_{A}^{2}\right)\right]-1\right)\right)$. Note that for the scenarios we are interested in (e.g., $v_{2} \ll v$ ) $m_{*}$ is smaller than its usual value in standard leptogenesis.

When only decays and inverse decays are considered, leptogenesis for given $K$ proceeds exactly as in standard hierarchical thermal leptogenesis (see, e.g., Ref. [63] for a review). In the weak washout regime $K \ll 1$, the baryon asymmetry strongly depends on the initial asymmetry and the initial $N_{1}$ abundance, with $N_{1}$ decays occurring at $T \ll M_{1}$. The strong washout regime $K \gg 1$ is independent of the initial conditions, and the asymmetry is generated as the $N_{1}$ fall out of thermal equilibrium.

The $2 \leftrightarrow 2$ scatterings with $\Delta L=1$ (see, e.g., Ref. [64]) provide a correction to the simple decays plus inverse decays picture; they act to increase $N_{1}$ production at $T>M_{1}$ and contribute to washout at $T<M_{1}$. In standard hierarchical thermal leptogenesis, the scattering contributions involving the top quark and the gauge bosons are roughly equal. In the present model, the gauge boson contribution is the same as in the standard scenario. However, by construction, the $\Phi_{2}$ involved here in leptogenesis does not couple directly to the top quark, and thus the usual s-channel $(N l \leftrightarrow t q)$ and t-channel $(N t \leftrightarrow l q, N q \leftrightarrow l t)$ scattering contributions do not occur. Instead, at large $\tan \beta$ they can be replaced by the analogous contribution from other charged fermions, i.e., the bottom quark in Type II and flipped $\nu 2 \mathrm{HDMs}$ and/or the tau lepton in Type II and LS $\nu 2 \mathrm{HDMs}$. A large tau lepton Yukawa will also introduce new s-channel $\left(N \Phi_{2} \leftrightarrow \tau \Phi_{2}\right)$ and t-channel $\left(N \Phi_{2} \leftrightarrow \tau \Phi_{2}, \tau N \leftrightarrow \Phi_{2} \bar{\Phi}_{2}\right)$ scattering contributions. All of these processes are proportional to $\left(y_{\nu}^{\dagger} y_{\nu}\right)_{11}$ and hence $M_{1} \tilde{m}_{1} / v^{2}$, with the appropriate $\nu 2 \mathrm{HDM}$ replacement for $v^{2}$. Therefore, they scale with the decays and inverse 
decays so that they represent only a minor (but obviously important) departure from the standard leptogenesis scenario.

The $2 \leftrightarrow 2$ scatterings with $\Delta L=2$ mediated by the right-handed neutrinos $\left(\Phi_{2} l \leftrightarrow \bar{\Phi}_{2} \bar{l}, \Phi_{2} \Phi_{2} \leftrightarrow l l\right)$ occur as they do in the standard scenario. These processes are proportional to $\operatorname{Tr}\left[\left(y_{\nu} y_{\nu}^{T}\right)\left(y_{\nu} y_{\nu}^{T}\right)^{\dagger}\right]$ and hence $M_{1}^{2} \bar{m}^{2} / v^{4}$ where $\bar{m}^{2}=\sum m_{i}^{2}$ is the neutrino mass scale $\gtrsim 0.05 \mathrm{eV}$. Comparing this rate to the decay/scattering rates $\propto M_{1} \tilde{m}_{1} / v^{2}$, it is easy to see that after making the appropriate $\nu 2 \mathrm{HDM}$ replacement for $v^{2}$, e.g., $v^{2} \rightarrow v_{2}^{2} \ll v^{2}$, these scatterings will become comparatively more important than in the standard case. For $T \lesssim$ $M_{1} / 3$ the thermally averaged $\Delta L=2$ scattering rate is well approximated by [63]

$$
\begin{aligned}
\frac{\Gamma_{\Delta L=2}}{H} \approx & \frac{T}{2.2 \times 10^{13} \mathrm{GeV}}\left(\frac{246 \mathrm{GeV}}{v}\right)^{4} \\
& \times\left(\frac{\bar{m}}{0.05 \mathrm{eV}}\right)^{2}
\end{aligned}
$$

where the previously described $\nu 2 \mathrm{HDM}$ replacements for $v^{2}$ hold (see Appendix B). In Fig. 5 (Fig. 6), we show the region in the $\lambda_{5}=0\left(m_{12}^{2}=0, \lambda_{5} \neq 0\right) \nu 2 \mathrm{HDM}$ where these scatterings are still in equilibrium at $T \lesssim M_{1} / 3$. $^{7}$ This is the region where strong $\Delta L=2$ scatterings can potentially wash out the generated asymmetry, depending on the details of the leptogenesis (e.g., in a weak washout scenario with $N_{1}$ decays at $T \ll M_{1}$, this washout may be avoided). Demanding that the scatterings fall out of equilibrium before sphaleron freeze-out at $T \sim 100 \mathrm{GeV}$ provides a lower bound $v_{2} \gtrsim 0.3$ or $\lambda_{5} \gtrsim 10^{-5}$; this is represented by the strong $\Delta L=2$ scattering washout regions in Figs. 5 and 6 , respectively. We note that this calculation has been performed in the context of a perturbative theory. This is reliable for the Type I $\nu 2 \mathrm{HDM}$ but not for Type II, LS, or flipped $\nu 2$ HDMs with sufficiently small $v_{2}$, when perturbativity breaks down.

Putting this all together, we can now read off from Figs. 5 and 6 the regions of parameter space which can achieve natural hierarchical thermal leptogenesis. For $\nu 2 \mathrm{HDMs}$ with $m_{12}^{2}>0$ and $\lambda_{5}=0$, we find $10^{3} \mathrm{GeV} \lesssim M_{N_{1}} \lesssim$ few $\times 10^{7} \mathrm{GeV}$ is viable for Type I $22 \mathrm{HDMs}$, and $10^{4} \mathrm{GeV} \lesssim M_{N_{1}} \lesssim$ few $\times 10^{7} \mathrm{GeV}$ for all other types if they are to remain perturbative. For the Ma model with

\footnotetext{
${ }^{7}$ A similar plot to Fig. 5 appears in Ref. [65] in the context of the Type I $\nu 2 \mathrm{HDM}$ with $v_{2}>0$. We are not aware of any plot similar to Fig. 6 in the literature, though see Refs. [66-70] for leptogenesis studies at points in the Ma model parameter space.
}

$m_{12}^{2}=0$ and $\lambda_{5} \neq 0$, we find viable parameter space for $10^{3} \mathrm{GeV} \lesssim M_{N_{1}} \lesssim 10^{8} \mathrm{GeV}$ and $10^{-5} \lesssim \lambda_{5} \lesssim 0.5$.

Lastly we note that the lightest scalar state in the Ma model is stable. ${ }^{8}$ It is therefore possible that this state, if it is neutral, constitutes some or all of the observed dark matter. During the leptogenesis epoch, $\Phi_{2}$ is produced in abundance in $N_{1}$ decays. Overproduction of dark matter is of no concern as long as $\Phi_{2}$ efficiently thermalizes at or below the temperatures when $N_{1}$ decays occur, which suggests $m_{22} \ll M_{N_{1}}$. In this case the lightest state is a thermal relic dark matter candidate.

\section{CONCLUSION}

The minimal Type I seesaw model is unable to explain neutrino masses and the BAU via hierarchical thermal leptogenesis without ceding naturalness. The main conclusion of this paper is the observation that a second Higgs doublet can avoid this problem. These $22 \mathrm{HDM}$ models provide a natural solution by reducing the (possibly effective) vev entering the seesaw formula. This can be done radiatively, or by having the second Higgs doublet provide a tree-level seesaw with a small vev $v_{2}$, kept natural by softly breaking a $U(1)$ or $Z_{2}$ symmetry.

The models naturally accommodate a SM-like Higgs and predict the existence of approximately $\mathrm{TeV}$-scale extra scalar states in order to remain natural. We rediscovered the radiative $\mathrm{Ma}$ model as the only possibility when $v_{2}=0$; in that case we found $10^{3} \mathrm{GeV} \lesssim M_{N_{1}} \lesssim 10^{8} \mathrm{GeV}$ and $10^{-5} \lesssim \lambda_{5} \lesssim 0.5$ could simultaneously explain neutrino masses and the BAU via leptogenesis while remaining natural. The $v_{2}>0$ models require $\tan \beta \gtrsim 4$; we found $10^{3} \mathrm{GeV} \lesssim M_{N_{1}} \lesssim$ few $\times 10^{7} \mathrm{GeV}$ was viable for Type I $\nu 2 \mathrm{HDMs}$, and $10^{4} \mathrm{GeV} \lesssim M_{N_{1}} \lesssim$ few $\times 10^{7} \mathrm{GeV}$ for all other types if they are to remain perturbative. The interesting areas of parameter space are well summarized in Figs. 5 and 6.

\section{ACKNOWLEDGMENTS}

This work was supported in part by the Australian Research Council.

\section{APPENDIX A: SQUARED SCALAR MASSES AT $\mathcal{O}\left(m_{12}^{4} / m_{22}^{4}\right)$}

To order $m_{12}^{4} / m_{22}^{4}$, the scalar masses Eqs. (14) are given by

\footnotetext{
${ }^{8}$ The lifetimes of the heavier scalar states are governed by mass splittings $\Delta$ via $\Gamma \sim G_{F}^{2} \Delta^{5} /\left(10^{2} \pi^{3}\right)$. In the parameter space of interest, one can check that $\Delta$ is typically already large enough at tree level so that lifetimes remain well below $\mathcal{O}(1 \mathrm{~s})$ and therefore do not disturb big bang nucleosynthesis.
} 


$$
\begin{aligned}
& m_{h}^{2} \approx v_{1}^{2}\left[\lambda_{1}+\frac{m_{12}^{4}}{m_{22}^{4}} \frac{2 \lambda_{345}-\lambda_{1}-\frac{v_{1}^{2}}{2 m_{22}^{2}} \lambda_{1} \lambda_{345}}{\left.\left(1+\frac{v_{1}^{2}}{2 m_{22}^{2}}\left(\lambda_{345}-2 \lambda_{1}\right)\right)\left(1+\frac{v_{1}^{2}}{2 m_{22}^{2}} \lambda_{345}\right)^{2}\right]}\right. \\
& m_{H}^{2} \approx m_{22}^{2}\left[1+\lambda_{345} \frac{v_{1}^{2}}{2 m_{22}^{2}}+\frac{m_{12}^{4}}{m_{22}^{4}} \frac{1-\frac{v_{1}^{2}}{2 m_{22}^{2}}\left(2 \lambda_{345}-3 \lambda_{2}\right)+\frac{v_{1}^{4}}{4 m_{22}^{4}}\left(\lambda_{345}^{2}+3 \lambda_{2} \lambda_{345}-6 \lambda_{1} \lambda_{2}\right)}{\left(1+\frac{v_{1}^{2}}{2 m_{22}^{2}}\left(\lambda_{345}-2 \lambda_{1}\right)\right)\left(1+\frac{v_{1}^{2}}{2 m_{22}^{2}} \lambda_{345}\right)^{2}}\right], \\
& m_{A}^{2} \approx m_{22}^{2}\left[1+\left(\lambda_{345}-2 \lambda_{5}\right) \frac{v_{1}^{2}}{2 m_{22}^{2}}+\frac{m_{12}^{4}}{m_{22}^{4}} \frac{1+\frac{v_{1}^{2}}{2 m_{22}^{2}}\left(\lambda_{345}+\lambda_{2}-2 \lambda_{5}\right)}{\left(1+\frac{v_{1}^{2}}{2 m_{22}^{2}} \lambda_{345}\right)^{2}}\right] \\
& m_{H^{ \pm}}^{2} \approx m_{22}^{2}\left[1+\lambda_{3} \frac{v_{1}^{2}}{2 m_{22}^{2}}+\frac{m_{12}^{4}}{m_{22}^{4}} \frac{1+\frac{v_{1}^{2}}{2 m_{22}^{2}}\left(\lambda_{2}+\lambda_{3}\right)}{\left(1+\frac{v_{1}^{2}}{2 m_{22}^{2}} \lambda_{345}\right)^{2}}\right] .
\end{aligned}
$$

\section{APPENDIX B: BOUNDS FOR MORE GENERAL $\boldsymbol{m}_{\nu}$}

We consider the seesaw Lagrangian as in Eq. (2) and a neutrino mass matrix of the form

$$
m_{\nu}=\frac{v^{2}}{2} y_{\nu} \mathcal{D}_{M}^{-1} \mathcal{D}_{f(M)} y_{\nu}^{T},
$$

where $\mathcal{D}_{x} \equiv \operatorname{diag}\left(x_{1}, x_{2}, x_{3}\right)$. Note that in the $\nu 2 \mathrm{HDM}$ with $\lambda_{5}=0$ we have $f\left(M_{j}\right)=v_{2}^{2} / v^{2}$, and in the Ma model with $M_{N} \gg m_{22}$, we have

$$
f\left(M_{j}\right)=\frac{\lambda_{5}}{8 \pi^{2}}\left(\ln \left[\frac{M_{j}^{2}}{\left(m_{H}^{2}+m_{A}^{2}\right) / 2}\right]-1\right) .
$$

Following Casas-Ibarra [71], it is possible to write

$$
y_{\nu}=\frac{\sqrt{2}}{v} U^{\dagger} \mathcal{D}_{m}^{\frac{1}{2}} R \mathcal{D}_{M}^{\frac{1}{2}} \mathcal{D}_{f(M)}^{-\frac{1}{2}},
$$

where $R$ is a (possibly complex) orthogonal $\left(R R^{T}=R^{T} R=\rrbracket\right)$ matrix. The Vissani bound on each right-handed neutrino mass becomes [17]

$$
\begin{aligned}
& \frac{1}{4 \pi^{2}} \frac{2}{v^{2}} \frac{M_{j}^{3}}{f\left(M_{j}\right)} \sum_{i} m_{i}\left|R_{i j}\right|^{2}<1 \mathrm{TeV}^{2} \\
& \Rightarrow M_{N_{1}} \lesssim 3 \times 10^{7} \mathrm{GeV} \times f\left(M_{N_{1}}\right)^{\frac{1}{3}} .
\end{aligned}
$$

The $C P$ asymmetry for hierarchical neutrinos [14] becomes

$$
\begin{aligned}
\left|\epsilon_{1}\right| & =\frac{6}{8 \pi} \frac{M_{1}}{v^{2}} \frac{\operatorname{Im}\left[\left(R^{\dagger} \mathcal{D}_{m}\left(R \mathcal{D}_{f(M)}^{-1} R^{T}\right) \mathcal{D}_{m} R^{*}\right)_{11}\right]}{\left(R^{\dagger} \mathcal{D}_{m} R\right)_{11}} \\
& \lesssim \frac{6}{8 \pi} \frac{m_{3} M_{N_{1}}}{v^{2}} \frac{1}{\min \left[f\left(M_{j}\right)\right]},
\end{aligned}
$$

where the approximate inequality holds for $\max \left(\left|R_{i j}\right|\right) \leq 1$. For larger $\max \left(\left|R_{i j}\right|\right)$ the inequality can be exceeded, but this corresponds to a fine-tuning (see Ref. [17]). With this caveat the Davidson-Ibarra bound for $\min \left[f\left(M_{i}\right)\right]=$ $f\left(M_{N_{1}}\right)$ therefore becomes

$$
M_{N_{1}} \gtrsim 5 \times 10^{8} \mathrm{GeV} \times f\left(M_{N_{1}}\right) .
$$

The $\Delta L=2$ scatterings are proportional to $[63,72]$

$$
\begin{aligned}
& \sum_{i, j} \operatorname{Re}\left[\left(y_{\nu}^{\dagger} y_{\nu}\right)_{i j}\left(y_{\nu}^{\dagger} y_{\nu}\right)_{i j}\right] \frac{1}{M_{i} M_{j}} \\
& =\operatorname{Tr}\left[\left(y_{\nu} \mathcal{D}_{M}^{-1} y_{\nu}^{T}\right)\left(y_{\nu} \mathcal{D}_{M}^{-1} y_{\nu}^{T}\right)^{\dagger}\right] \\
& =\frac{4}{v^{4}} \operatorname{Tr}\left[\mathcal{D}_{m}\left(R \mathcal{D}_{f(M)}^{-1} R^{T}\right) \mathcal{D}_{m}\left(R \mathcal{D}_{f(M)}^{-1} R^{T}\right)^{\dagger}\right] \\
& \lesssim \frac{4}{v^{4}} \frac{\bar{m}^{2}}{\min \left[f\left(M_{j}\right)\right]^{2}},
\end{aligned}
$$

where the last line is an exact equality for $f\left(M_{1}\right)=f\left(M_{2}\right)=f\left(M_{3}\right)$, an exact inequality when $R$ is real, and an approximate inequality (as indicated) if $R$ is complex with $\max \left(\left|R_{i j}\right|\right) \leq 1$. Again, for larger $\max \left(\left|R_{i j}\right|\right)$ the inequality can be exceeded. Then for $\min \left[f\left(M_{i}\right)\right]=$ $f\left(M_{N_{1}}\right)$ the $\Delta L=2$ scattering Eq. (35) becomes

$$
\frac{\Gamma_{\Delta L=2}}{H} \lesssim \frac{T}{2.2 \times 10^{13} \mathrm{GeV}} \frac{1}{f\left(M_{N_{1}}\right)^{2}}\left(\frac{\bar{m}}{0.05 \mathrm{eV}}\right)^{2}
$$


[1] G. Aad et al. (ATLAS Collaboration), Observation of a new particle in the search for the Standard Model Higgs boson with the ATLAS detector at the LHC, Phys. Lett. B 716, 1 (2012).

[2] S. Chatrchyan et al. (CMS Collaboration), Observation of a new boson at a mass of $125 \mathrm{GeV}$ with the CMS experiment at the LHC, Phys. Lett. B 716, 30 (2012).

[3] B. T. Cleveland, T. Daily, R. Davis, Jr., J. R. Distel, K. Lande, C. K. Lee, P. S. Wildenhain, and J. Ullman, Measurement of the solar electron neutrino flux with the Homestake chlorine detector, Astrophys. J. 496, 505 (1998).

[4] Y. Fukuda et al. (Super-Kamiokande Collaboration), Evidence for Oscillation of Atmospheric Neutrinos, Phys. Rev. Lett. 81, 1562 (1998).

[5] W. Hampel et al. (GALLEX Collaboration), GALLEX solar neutrino observations: Results for GALLEX IV, Phys. Lett. B 447, 127 (1999).

[6] Q. R. Ahmad et al. (SNO Collaboration), Measurement of the Rate of $\nu_{e}+d \rightarrow p+p+e^{-}$Interactions Produced by ${ }^{8} B$ Solar Neutrinos at the Sudbury Neutrino Observatory, Phys. Rev. Lett. 87, 071301 (2001).

[7] Q. R. Ahmad et al. (SNO Collaboration), Direct Evidence for Neutrino Flavor Transformation from Neutral Current Interactions in the Sudbury Neutrino Observatory, Phys. Rev. Lett. 89, 011301 (2002).

[8] K. Eguchi et al. (KamLAND Collaboration), First Results from KamLAND: Evidence for Reactor Anti-Neutrino Disappearance, Phys. Rev. Lett. 90, 021802 (2003).

[9] P. Minkowski, $\mu \rightarrow e \gamma$ at a rate of one out of 1-billion muon decays?, Phys. Lett. B 67, 421 (1977).

[10] R. N. Mohapatra and G. Senjanovic, Neutrino Mass and Spontaneous Parity Violation, Phys. Rev. Lett. 44, 912 (1980).

[11] T. Yanagida, Horizontal symmetry and masses of neutrinos, Conf.Proc. C7902131, 95 (1979).

[12] M. Gell-Mann, P. Ramond, and R. Slansky, Complex spinors and unified theories, Conf.Proc. C790927, 315 (1979).

[13] M. Fukugita and T. Yanagida, Baryogenesis without grand unification, Phys. Lett. B 174, 45 (1986).

[14] S. Davidson and A. Ibarra, A lower bound on the righthanded neutrino mass from leptogenesis, Phys. Lett. B 535, 25 (2002).

[15] G. F. Giudice, A. Notari, M. Raidal, A. Riotto, and A. Strumia, Towards a complete theory of thermal leptogenesis in the SM and MSSM, Nucl. Phys. B685, 89 (2004).

[16] F. Vissani, Do experiments suggest a hierarchy problem?, Phys. Rev. D 57, 7027 (1998).

[17] J. D. Clarke, R. Foot, and R. R. Volkas, Electroweak naturalness in three-flavour Type I see-saw and implications for leptogenesis, Phys. Rev. D 91, 073009 (2015).

[18] F. Bazzocchi and M. Fabbrichesi, Little hierarchy problem for new physics just beyond the LHC, Phys. Rev. D 87, 036001 (2013).

[19] M. Fabbrichesi and A. Urbano, Naturalness redux, Phys. Rev. D 92, 015028 (2015).

[20] A. Pilaftsis and T. E. J. Underwood, Resonant leptogenesis, Nucl. Phys. B692, 303 (2004).
[21] E. K. Akhmedov, V. A. Rubakov, and A. Yu. Smirnov, Baryogenesis via Neutrino Oscillations, Phys. Rev. Lett. 81, 1359 (1998).

[22] E. Ma, Verifiable radiative seesaw mechanism of neutrino mass and dark matter, Phys. Rev. D 73, 077301 (2006).

[23] G. C. Branco, P. M. Ferreira, L. Lavoura, M. N. Rebelo, M. Sher, and J. P. Silva, Theory and phenomenology of twoHiggs-doublet models, Phys. Rep. 516, 1 (2012).

[24] E. A. Paschos, Diagonal neutral currents, Phys. Rev. D 15, 1966 (1977).

[25] S. L. Glashow and S. Weinberg, Natural conservation laws for neutral currents, Phys. Rev. D 15, 1958 (1977).

[26] E. Ma, Naturally Small Seesaw Neutrino Mass with No New Physics Beyond the TeV Scale, Phys. Rev. Lett. 86, 2502 (2001).

[27] F. Wang, W. Wang, and J. M. Yang, Split two-Higgs-doublet model and neutrino condensation, Europhys. Lett. 76, 388-394 (2006).

[28] S. Gabriel and S. Nandi, A new two Higgs doublet model, Phys. Lett. B 655, 141 (2007).

[29] S. M. Davidson and H. E. Logan, Dirac neutrinos from a second Higgs doublet, Phys. Rev. D 80, 095008 (2009).

[30] J. F. Gunion and H. E. Haber, The $C P$ conserving two Higgs doublet model: The approach to the decoupling limit, Phys. Rev. D 67, 075019 (2003).

[31] N. G. Deshpande and E. Ma, Pattern of symmetry breaking with two Higgs doublets, Phys. Rev. D 18, 2574 (1978).

[32] K. G. Klimenko, On necessary and sufficient conditions for some Higgs potentials to be bounded from below, Theor. Math. Phys. 62, 58 (1985).

[33] M. Maniatis, A. von Manteuffel, O. Nachtmann, and F. Nagel, Stability and symmetry breaking in the general two-Higgs-doublet model, Eur. Phys. J. C 48, 805 (2006).

[34] A. Barroso, P. M. Ferreira, I. P. Ivanov, and R. Santos, Metastability bounds on the two Higgs doublet model, J. High Energy Phys. 06 (2013) 045.

[35] S. Kanemura, T. Kubota, and E. Takasugi, Lee-QuiggThacker bounds for Higgs boson masses in a two doublet model, Phys. Lett. B 313, 155 (1993).

[36] A. G. Akeroyd, A. Arhrib, and E.-M. Naimi, Note on tree level unitarity in the general two Higgs doublet model, Phys. Lett. B 490, 119 (2000).

[37] I. F. Ginzburg and I. P. Ivanov, Tree-level unitarity constraints in the most general 2HDM, Phys. Rev. D 72, 115010 (2005).

[38] U. Nierste and K. Riesselmann, Higgs sector renormalization group in the MS and OMS scheme: The breakdown of perturbation theory for a heavy Higgs, Phys. Rev. D 53, 6638 (1996).

[39] N. Chakrabarty, U. K. Dey, and B. Mukhopadhyaya, Highscale validity of a two-Higgs doublet scenario: a study including LHC data, J. High Energy Phys. 12 (2014) 166.

[40] D. Das and I. Saha, Search for a stable alignment limit in two-Higgs-doublet models, Phys. Rev. D 91, 095024 (2015).

[41] D. Chowdhury and O. Eberhardt, arXiv:1503.08216.

[42] J. Bijnens, J. Lu, and J. Rathsman, Constraining general two Higgs doublet models by the evolution of Yukawa couplings, J. High Energy Phys. 05 (2012) 118. 
[43] C.-Y. Chen, S. Dawson, and M. Sher, Heavy Higgs searches and constraints on two Higgs doublet models, Phys. Rev. D 88, 015018 (2013).

[44] O. Eberhardt, U. Nierste, and M. Wiebusch, Status of the two-Higgs-doublet model of type II, J. High Energy Phys. 07 (2013) 118.

[45] N. Craig, J. Galloway, and S. Thomas, arXiv:1305.2424.

[46] G. Belanger, B. Dumont, U. Ellwanger, J. F. Gunion, and S. Kraml, Global fit to Higgs signal strengths and couplings and implications for extended Higgs sectors, Phys. Rev. D 88, 075008 (2013).

[47] S. Chang, S. K. Kang, J.-P. Lee, K. Y. Lee, S. C. Park, and J. Song, Two Higgs doublet models for the LHC Higgs boson data at $\sqrt{s}=7$ and $8 \mathrm{TeV}$, J. High Energy Phys. 09 (2014) 101.

[48] B. Dumont, J. F. Gunion, Y. Jiang, and S. Kraml, Constraints on and future prospects for two-Higgs-doublet models in light of the LHC Higgs signal, Phys. Rev. D 90, 035021 (2014).

[49] Y. Amhis et al. (Heavy Flavor Averaging Group Collaboration), arXiv:1412.7515.

[50] M. Misiak et al., Updated NNLO QCD Predictions for the Weak Radiative B-Meson Decays, Phys. Rev. Lett. 114, 221801 (2015).

[51] T. Junk et al. (LEP Higgs Working Group for Higgs boson searches, ALEPH, DELPHI, L3, OPAL Collaborations), arXiv:hep-ex/0107031.

[52] G. Aad et al. (ATLAS Collaboration), Search for neutral Higgs bosons of the minimal supersymmetric standard model in pp collisions at $\sqrt{s}=8 \mathrm{TeV}$ with the ATLAS detector, J. High Energy Phys. 11 (2014) 056.

[53] V. Khachatryan et al. (CMS Collaboration), Search for neutral MSSM Higgs bosons decaying to a pair of tau leptons in pp collisions, J. High Energy Phys. 10 (2014) 160.

[54] G. Aad et al. (ATLAS Collaboration), Search for charged Higgs bosons decaying via $H^{ \pm} \rightarrow \tau^{ \pm} \nu$ in fully hadronic final states using $p p$ collision data at $\sqrt{s}=8 \mathrm{TeV}$ with the ATLAS detector, J. High Energy Phys. 03 (2015) 088.

[55] CMS Collaboration, Tech. Rep. CMS-PAS-HIG-14-020, 2014.
[56] T. Toma and A. Vicente, Lepton flavor violation in the scotogenic model, J. High Energy Phys. 01 (2014) 160.

[57] J. R. Ellis, K. Enqvist, D. V. Nanopoulos, and F. Zwirner, Observables in low-energy superstring models, Mod. Phys. Lett. A 01, 57 (1986).

[58] R. Barbieri and G. F. Giudice, Upper bounds on supersymmetric particle masses, Nucl. Phys. B306, 63 (1988).

[59] B. Grzadkowski, M. Lindner, and S. Theisen, Nonlinear evolution of Yukawa couplings in the double Higgs and supersymmetric extensions of the Standard Model, Phys. Lett. B 198, 64 (1987).

[60] A. Merle and M. Platscher, arXiv:1502.03098.

[61] R. Foot, A. Kobakhidze, K. L. McDonald, and R. R. Volkas, Poincare protection for a natural electroweak scale, Phys. Rev. D 89, 115018 (2014).

[62] H. Davoudiasl and I. M. Lewis, Right-handed neutrinos as the origin of the electroweak scale, Phys. Rev. D 90, 033003 (2014).

[63] W. Buchmuller, P. Di Bari, and M. Plumacher, Leptogenesis for pedestrians, Ann. Phys. (Amsterdam) 315, 305 (2005).

[64] S. Davidson, E. Nardi, and Y. Nir, Leptogenesis, Phys. Rep. 466, 105 (2008).

[65] N. Haba and O. Seto, Low scale thermal leptogenesis in neutrinophilic Higgs doublet models, Prog. Theor. Phys. 125, 1155 (2011).

[66] E. Ma, Common origin of neutrino mass, dark matter, and baryogenesis, Mod. Phys. Lett. A 21, 1777 (2006).

[67] T. Hambye, F.-S. Ling, L. Lopez Honorez, and J. Rocher, Scalar multiplet dark matter, J. High Energy Phys. 07 (2009) 090.

[68] D. Suematsu, Thermal leptogenesis in a TeV scale model for neutrino masses, Eur. Phys. J. C 72, 1951 (2012).

[69] S. Kashiwase and D. Suematsu, Baryon number asymmetry and dark matter in the neutrino mass model with an inert doublet, Phys. Rev. D 86, 053001 (2012).

[70] J. Racker, Mass bounds for baryogenesis from particle decays and the inert doublet model, J. Cosmol. Astropart. Phys. 03 (2014) 025.

[71] J. A. Casas and A. Ibarra, Oscillating neutrinos and $\mu \rightarrow e \gamma$, Nucl. Phys. B618, 171 (2001).

[72] W. Buchmuller and M. Plumacher, $C P$ asymmetry in Majorana neutrino decays, Phys. Lett. B 431, 354 (1998). 


\section{University Library}

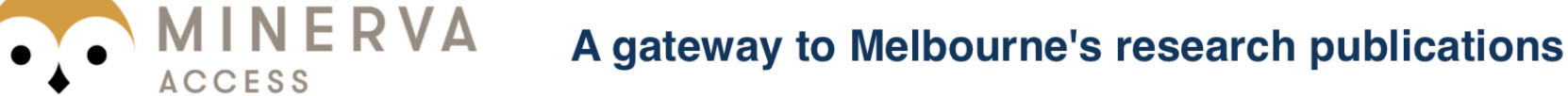

Minerva Access is the Institutional Repository of The University of Melbourne

Author/s:

Clarke, JD;Foot, R;Volkas, RR

Title:

Natural leptogenesis and neutrino masses with two Higgs doublets

Date:

2015-08-17

Citation:

Clarke, J. D., Foot, R. \& Volkas, R. R. (2015). Natural leptogenesis and neutrino

masses with two Higgs doublets. PHYSICAL REVIEW D, 92 (3), https://doi.org/10.1103/ PhysRevD.92.033006.

Persistent Link:

http://hdl.handle.net/11343/115946 